\title{
AN H1 -GALERKIN METHOD FOR A STEFAN PROBLEM WITH A QUASILINEAR PARABOLIC EQUATION IN NON-DIVERGENCE FORM
}

\author{
A.K. PANI \\ and \\ P.C. DAS \\ Department of Mathematics \\ I.I.T., KANPUR - 208016, India \\ (Received December 10, 1985)
}

ABSTRACT. Optimal error estimates in $\mathrm{L}^{2}, \mathrm{H}^{1}$ and $\mathrm{H}^{2}$-norms are established for a single phase stefan problem with quasilinear parabolic equation in non-divergence form by an $H^{1}-$ Galerkin procedure.

LEY WORDS AND PHRASES. $\mathrm{H}^{1}$-Galerkin procedure, finite element approximation, nonlinear Stefan problem, non-divergence form, error analysis.

1980 ays SUBJECT Classification CODE. $65 \mathrm{~N} 15,65 \mathrm{~N} 30$.

\section{IRTRODUCTION.}

With the help of Galerkin finite element methods, Nitsche in his pioneering works [1]-[3] established error estimates for linear problems, proposed earlier by Magenes [4]. We extended his analysis to nonlinear problems in divergence form [5]-[6]. In the present work, a single phase stefan problem with quasilinear parabolic equation in non-divergence form is considered and under appropriate conditions optimal error estimates for Galerkin approximation in $\mathrm{L}^{2}, \mathrm{H}^{1}$ as well as $\mathrm{H}^{2}$ norms are established. We require more regularity assumptions for the present one than for the cases discussed in [5]-[6], and consequently we improve upon the estimates in $L^{2}$-norm.

The organization of the paper is as follows: In section 2, the description of the problem and the transformed system with some preliminaries are presented. The weak formulation and $\mathrm{H}^{1}$-Galerkin procedure are discussed in section 3 . Section 4 deals with an auxiliary projection and some approximation Lemmas. In section 5 optimal error estimates in $\mathrm{L}^{2}, \mathrm{H}^{1}$ and $\mathrm{H}^{2}$-norms for continuous time Galerkin approximations are established, assuming existence of the approximate solution. Finally, in section 6 the question of global existence and uniqueness of the Galerkin approximation is discussed.

\section{PROBLEM DESCRIPTION AND DOMAIN FIXING.}

The nonlinear heat conduction with change of phase can be modelled as a single phase nonlinear Stefan problem in a variable domain $\Omega(\tau) \times\left(0, \mathrm{~T}_{0}\right)$, where $\Omega(\tau)=$ $\{y \cdot \varepsilon(0, S(\tau))$ and $S(\tau)$ known to be the free boundary. We state this problem as follows:

Find a pair $\{U, S\}, U=U(y, \tau), S=S(\tau)$ such that $U$ satisfies 


$$
U_{\tau}-a(U) U_{y y}=0, \text { for }(y, \tau) \varepsilon \Omega(\tau)<\left(0, T_{0}\right]
$$

with initial and boundary conditions

$$
\begin{array}{ll}
U(y, 0)=g(y), & \text { for } y \in I=(0,1) \\
U_{y}(0, . \tau)=0 & \\
\text { for } \pi>0 & \text { for } \tau>0 \\
U(S(\tau), \tau)=0, &
\end{array}
$$

and $S$, the free boundary satisfies

$$
S_{\tau}=-U_{y}(S(\tau), \tau), \quad \text { for } \tau>0
$$

with $\mathrm{S}(0)=1$. The above problems is a special case of the general situation discussed in Fasano et. a1. [7], where ' $a$ ' depends only on $U, q \equiv 0$ and $\phi=-U y(S(\tau), \tau)$ in their notations.

We use the following notiations. Let $\Omega(\tau)$ R be a bounded domain for $\tau \geq 0$. Let $(u, v)=\int_{\Omega(\tau)} u v d x$ and $\|u\|^{2}=(u, u)$. For each nonnegative integer m, let $H^{m}(\Omega(\tau))$ be the usual Sobolev space $w^{m, P}(\Omega(\tau))$, for $p=2$ with the norm

$$
\|u\|_{H^{m}(\Omega(\tau))}^{2}=\sum_{j=0}^{m}\left\|\frac{\partial^{j}}{\partial x^{j}} u(x, \tau)\right\|^{2} d x .
$$

Further, $W^{m}, \infty(\Omega(\tau))$ is defined as usual with the norm

$$
\|u\|_{W^{m}, \infty(\Omega(\tau))}=\sum_{j=0}^{m}\left\|\frac{\partial^{j} u}{\partial x^{j}}\right\|_{L}^{\infty}(\Omega(\tau)) .
$$

In case $I=\Omega(\tau)$, we shall omit $I$ from $H^{m}(I), L^{\infty}(I)$ and $W^{m, \infty}(I)$ and norm $H^{m}(I)$ is denoted by $\|\cdot\|_{m}$.

If $X$ be a normed linear space with norm $\|\cdot\|_{X}$ and $\phi:(a, b) \rightarrow X$, then we denote by

$$
\|\phi\|_{W}^{q}, q(a, b ; x)=\sum_{\beta=0}^{k}\left\|\frac{\partial^{\beta} \phi}{\partial t^{\beta}}\right\|_{L^{q}(a, b ; x)}^{q}, 1 \leq q<\infty
$$

and

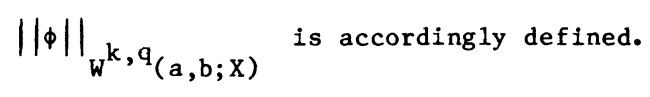


In case $(a, b)=(0, T)$ and $X=H^{m}$ or $W^{m, \infty}$, we write simply $\left.\|\phi\|_{W^{k, q}} H^{m}\right)$

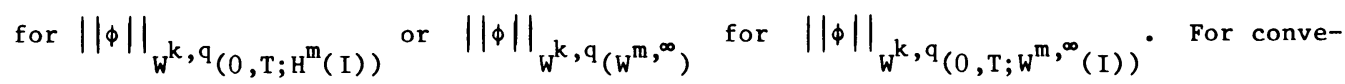
nience, we use $\phi_{x}=\frac{\partial \phi}{\partial x}, \phi_{x x}=\frac{\partial^{2} \phi}{\partial x^{2}} ; \phi_{t}=\frac{\partial \phi}{\partial t}$ and $\phi(1)=\phi(1, t)$, if $\phi=\phi(x, t)$.

Throughout this work, $\mathrm{K}$ will always denote a generic constant. On occasion, we will show that a constant depends on certain parameters, while independent of others.

We shall now state our main assumption on $\left.\mathrm{a}()_{\cdot}\right), \mathrm{g}$ and the solution $\mathrm{U,S}$, and call them collectively 'condition B'.

\section{CONDITION B.}

(i) For $p \in R, a(p) \geq \alpha$, where $\alpha$ is a positive constant.

(ii) For $p \in R, a(p) \varepsilon c^{3}(R)$ and there is a common bound $k_{1}>0$ such that $|a|$, $\left|a_{p}\right|,\left|a_{p p}\right|$ and $\left|a_{p p p}\right| \leq K_{1}$.

(iii) The initial function $g$ is sufficiently smooth and satisfies the compatibility condition that is $g_{y}(0)=g(1)=0$.

(iv) The problem (2.1)-(2.4) has a unique solution.

For the existence and uniqueness of the solution of (2.1)-(2.4), see Fasano et.a1. [7].

Further it is assumed that the solution U,S of (2.1)-(2.4) satisfies the following regularity condition. For an integer $r \geq 1$,

$$
\begin{aligned}
& \mathrm{U} \varepsilon \mathrm{L}^{\infty}\left(0, \mathrm{~T}_{0} ; \mathrm{H}^{\mathrm{r}+1}(\Omega(\tau)) \quad \mathrm{W}^{1,2}\left(0, \mathrm{~T}_{0} ; \mathrm{H}^{\mathrm{r}+1}(\Omega(\tau))\right) \cap \mathrm{W}^{1, \infty}\left(0, \mathrm{~T}_{0} ; \mathrm{W}^{2, \infty}(\Omega(\tau))\right),\right. \\
& \mathrm{S} \varepsilon \mathrm{W}^{1, \infty}\left(0, \mathrm{~T}_{0}\right) .
\end{aligned}
$$

Let $\tilde{\mathrm{K}}_{2}$ be the bound for the functions in above mentioned norms.

We fix the free boundary, using Landau type transformation [8]

$$
\mathrm{x}=\mathrm{s}^{-1}(\tau) \mathrm{y}, \quad \tau \geq 0
$$

Further, we introduce an additional transformation in time scale given by

$$
t=t(\tau)=\int_{0}^{\tau} S^{-2}\left(\tau^{\prime}\right) d \tau^{\prime}
$$

in order to decouple the resulting transformed system. A routine calculation shows that the function $u(x, t)=U(y, \tau)$ satisfies

$$
\begin{gathered}
u_{t}-a(u) u_{x x}=-u_{x}(1) x u_{x}, x \varepsilon I, t \varepsilon(0, T] \\
u(x, 0)=g(x), x \varepsilon I ; \\
u_{x}(0, t)=u(1, t)=0, t>0
\end{gathered}
$$


and the function $s(t)=S(\tau)$ satisfies

$$
\frac{d s}{d t}=-u_{x}(1) s, t>0
$$

with $\mathbf{s}(0)=1$.

Here, $t=\mathrm{T}$ corresponds to $\tau=\mathrm{T}_{0}$. Note that all the regularity assumptions for $\mathrm{U}, \mathrm{S}$ are carried over to $\mathrm{u}, \mathrm{s}$ with the bound say $\mathrm{K}_{2}$ and the new regularity assumptions are collectively called $R_{1}$. Further, the integral (2.6) can be rewritten as

$$
\frac{d \tau}{d t}=s^{2}(t), \quad \text { with } \quad \tau(0)=0
$$

\section{WEAK FORHLLATION AND $\mathbf{H}^{\mathbf{l}}$-GaLERKIN PROCEDURE.}

Consider the space:

$$
\mathrm{H}^{2}(\mathrm{I})=\left\{\mathrm{v} \in \mathrm{H}^{2}(\mathrm{I}): \mathrm{v}_{\mathrm{x}}(0)=\mathrm{v}(1)=0\right\},
$$

The weak formulation of $(2.7)-(2.9)$ is given by

$$
\left(u_{t x}, v_{x}\right)+\left(a(u) u_{x x}, v_{x x}\right)=u_{x}(1)\left(x_{x}, v_{x x}\right), v \in \stackrel{0}{H}^{2}(I) \text { and } t>0
$$

with $u(x, 0)=g(x)$.

$H^{1-G a l e r k i n ~ P r o c e d u r e . ~ L e t ~} S_{h}(0<h \leq 1)$ be a finite dimensional subspace of ${ }_{\mathrm{H}}^{2}(\mathrm{I})$ belonging to regular $\mathrm{S}_{\mathrm{h}}^{0 \mathrm{r}, 2}$ family, for a definition see oden et. al. [9] and satisfying the following approximation and inverse properties:

(i) For $v \in \mathrm{H}^{\mathrm{m}}(\mathrm{I}) \quad \mathrm{H}^{2}(\mathrm{I})$, there is a constant $\mathrm{K}_{0}$ independent of $\mathrm{h}$ such that

$$
\underset{x \in S_{h}}{\inf }\|v-x\|_{j} \leq k_{0} h^{m-j}\|v\|_{m} \text {, for } j=0,1,2 \text { and } 2 \leq m \leq r+1 ;
$$

(ii) For $x \in \stackrel{0}{s}_{h}, \quad\|x\|_{2} \leq \mathrm{k}_{0} \mathrm{~h}^{-1}\|x\|_{1}$.

Now we call $u^{h}:(0, T] \rightarrow \stackrel{S}{S}_{h}$ an $H^{l}$-Galerkin approximation of $u$, if it satisfies

$$
\left(u_{t x}^{h}, x_{x}\right)+\left(a\left(u^{h}\right) u_{x x}^{h}, x_{x x}\right)=u_{x}^{h}(1)\left(x u_{x}^{h}, x_{x x}\right), x \varepsilon s_{h}^{0}
$$

and the initial condition

$$
u^{h}(x, 0)=Q_{h} g(x)
$$

where $Q_{h}$ is an appropriate projection of $u$ onto $S_{h}$ at $t=0$, to be defined later. Further, the Galerkin approximations $s_{h}$ and $\tau_{h}$ of $s$ and $\tau$ respectively are given by 


$$
\frac{d s_{h}}{d t}=-u_{x}^{h}(1) s_{h}, \quad \text { with } \quad s_{h}(0)=1
$$

and

$$
\frac{d \tau}{d t}=s_{h}^{2}, \quad \text { with } \tau_{h}(0)=0
$$

\section{SOME APPROXIMATION LEMMAS.}

Set

$$
A(u ; v, w)=\left(a(u) v_{x x}, w_{x x}\right)-u_{x}(1)\left(x v_{x}, w_{x x}\right) \text {; for } u \varepsilon w^{1, \infty}, v \text { and } w \varepsilon H^{2} \text {. }
$$

The boundedness and Garding type inequality for $A$ can be established by standard arguments.

LEMMA 4.1. For $\mathrm{u} \varepsilon \mathrm{w}^{1, \infty}, \mathrm{v}$ and $\mathrm{w} \in \mathrm{H}^{2}(\mathrm{I})$

$$
|A(u ; v, w)| \leq M|| v_{x x}\left\||| w_{x x}\right\|
$$

and

$$
\mathrm{A}(\mathrm{u} ; \mathrm{v}, \mathrm{v}) \geq \tilde{\alpha}\left\|\mathrm{v}_{\mathrm{xx}}\right\|^{2}-\rho\left\|\mathrm{v}_{\mathrm{x}}\right\|^{2}
$$

where $M, \tilde{\alpha}$ and $\rho$ are constants, but $M$ and $\rho$ may depend on $\left\|u_{x}\right\|_{L}$.
Define

$$
A_{\rho}(u ; v, w)=A(u ; v, w)+\rho\left(v_{x}, w_{x}\right)
$$

Note that $A_{p}(u, .,$.$) is coercive in \mathrm{H}^{2}$, that is

$$
A_{\rho}(u ; v, v) \geq \tilde{\alpha}\left\|v_{x x}\right\|^{2}
$$

Let $\tilde{u} \varepsilon S_{h}$ be an approximation of $u$ with respect to the form $A_{\rho}$ :

$$
A_{\rho}(u ; u-\tilde{u}, x)=0, \quad x \in \stackrel{0}{S}_{h},
$$

Now, an application of Lax-Milgram theorem shows the existence of a unique solution $\tilde{u}$ of equation (4.5).

Consider

$$
L *(u) \phi=\left(a(u) \phi_{x x}\right)_{x x}+u_{x}(1)\left(x \phi_{x x}\right)_{x}-\rho \phi_{x x}, u \varepsilon \stackrel{0}{H^{2}}
$$

For $\Psi \varepsilon \mathrm{L}^{2}(\mathrm{I})$, define $\phi \varepsilon \mathrm{H}^{4} \quad \mathrm{H}^{2}$ by

$$
\begin{aligned}
& L^{*}(u) \phi=\Psi ; \quad x \in I \\
& \left.\phi_{x x}\right|_{x=1}=\left.\phi_{x x x}\right|_{x=0}=0 .
\end{aligned}
$$


Then, for $v \in \mathrm{H}^{2}(\mathrm{I})$ we get

$$
\left(v, L^{*}(u) \phi\right)=A_{\rho}(u ; v, \phi) .
$$

Thus, defining $D\left(L^{*}\right)$ as

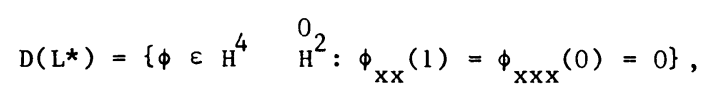

we have from the positivity and boundedness of $A_{\rho}$ that at least a weak solution $\phi \varepsilon D\left(L^{\star}\right)$ of (4.7) for each $\Psi \varepsilon L^{2}$ exists and the regularity

$$
\|\phi\|_{4} \leq c_{0}\|\Psi\|
$$

where $C_{0}$ depends on $u$ and its derivatives, holds.

Let $n=u-\tilde{u}$. We now need to obtain some estimates of $n$ and its temporal derivatives $n_{t}$, for our future use. The following Lemma proves very convenient for our purpose.

LEMMA 4.2. Let $\$ \varepsilon \mathrm{H}^{2}(\mathrm{I})$ and satisfy

$$
A_{\rho}(u ; p, x)=F(x), x \in S_{h}^{0}
$$

where $F: \stackrel{\mathrm{O}_{2}}{\mathrm{H}}(\mathrm{I}) \rightarrow \mathrm{R}$ and 1inear. Let there exist constants $M_{1}$ and $M_{2}$ with $M_{1} \geq M_{2}$ such that

$$
|F(\phi)| \leq M_{1}|| \phi_{x x} \|, \quad \phi \varepsilon H^{2}
$$

and

$$
|F(\phi)| \leq M_{2}\|\phi\|_{4}, \quad \phi \varepsilon D\left(L^{*}\right)
$$

Then, for sufficiently small h

$$
\left\|\Phi_{x x}\right\| \leq k_{3}\left[M_{1}+\inf \underset{0}{\|}\left\|s_{h}-x\right\|_{2}\right]
$$

and

$$
\begin{gathered}
\|D\| \leq K_{3}\left[(M_{1}+\inf \underbrace{}_{0}||-x \|_{2}) h^{2}+M_{2}\right], \\
x \in S_{h}
\end{gathered}
$$

where $K_{3}=K_{3}\left(\alpha, \rho, M, C_{0} ; K_{0}\right)$ is used as generic constant.

PROOF. Note that

$$
A_{\rho}(u ; \Phi, \Phi)=A_{\rho}(u ; \Phi, \Phi-x)-F(\Phi-x)+F(\Phi), x \in S_{h}^{0}
$$

By coercive property (4.4) for $A_{\rho}$, we get

$$
\begin{gathered}
\left\|\Phi_{x \mathbf{x}}\right\|^{2} \leq(\tilde{\alpha})^{-1}\left[\left(M \mid \phi_{x \mathbf{x}} \|+M_{1}\right)\right. \\
\left.\inf \|\$-x\|_{2}+M_{1}\left\|\Phi_{x x}\right\|\right] \\
x \in S_{h}
\end{gathered}
$$


For $\varepsilon \mathrm{H}^{0},\|\otimes\|_{2} \leq \mathrm{x}\left\|\Phi_{\mathrm{xx}}\right\|$. Therefore,

$$
\begin{gathered}
\left\|\Phi_{x \mathbf{x}}\right\| \leq(\tilde{\alpha})^{-1}\left[M \text { inf }\|\neq-x\|_{2}+2 K M_{1}\right] \\
x \in S_{h}
\end{gathered}
$$

and the estimate (4.13) follows. In order to get an $\mathrm{L}^{2}$-estimate, we follow here Aubin-Nitsche duality arguments. For $\Psi \varepsilon \mathrm{L}^{2}(\mathrm{I})$, define $\phi \varepsilon D\left(L^{*}\right)$ by (4.7). Multiply both the sides of $(4.7)$ by $\downarrow$ to obtain for $u \in \mathrm{H}^{2}$,

$$
\begin{aligned}
& (\Phi, \Psi)=\left(\phi, L^{*}(u) \phi\right)=A(u ; \Phi, \phi) \\
& =A(u ; \phi, \phi-x)+F(x-\phi)+F(\phi) \\
& \leq\left[M\left\|_{x x}\right\| \text { inf }\|\phi-x\|_{2}+M_{1} \text { inf }\|\phi-x\|_{2}\right]+M_{2}\|\phi\|_{4} \\
& x \in S_{h} \quad x \in S_{h} \\
& \leq\left[M\|\|_{x x} \| \mathrm{K}_{0} h^{2}+M_{1} K_{0} h^{2}+M_{2}\right]\|\phi\| \|_{4} .
\end{aligned}
$$

From (4.9), (4.13) and (4.15), we obtain the required estimate (4.14).

The next Lemma contains the error estimates related to $n$ and $\eta_{t}$.

Lema 4.3. For $t \in[0, T]$, the following estimates

$$
\|n\|_{j} \leq k_{4} h^{m-j}\|u\|_{m}
$$

and

$$
\begin{array}{r}
\left\|n_{t}\right\|_{j} \leq k_{5} h^{m-j}\left(\|u\|_{m}+\left\|u_{t}\right\|_{m}\right), \\
j=0,1,2 \text { and } 2 \leq m \leq r+1,
\end{array}
$$

hold. Here $\mathrm{K}_{4}$ and $\mathrm{K}_{5}$ are positive constants depending on parameters expressed through the following expressions that is

$$
\mathrm{K}_{4}=\mathrm{K}_{4}\left(\mathrm{~K}_{0}, \mathrm{~K}_{3}\right) \text { and } \mathrm{K}_{5}=\mathrm{K}_{5}\left(\mathrm{~K}_{0}, \mathrm{~K}_{1}, \mathrm{~K}_{3}, \mathrm{~K}_{4},\left\|\mathrm{u}_{\mathrm{t}}\right\|_{\mathrm{w}} 2, \infty \text { and }\|\mathrm{u}\|_{\mathrm{w}^{2, \infty}}\right) \text {. }
$$

PRO0F. Put $\Phi=\eta$ and $F=0$ in the previous Lemma 4.2 to get

$$
\begin{aligned}
&\left\|n_{x x}\right\| \leq k_{3} \text { inf }\|n-x\|_{2} \\
& \quad x \in s_{h} \\
& \leq k_{3} \quad \text { inf }\|u-v\|_{2}, v=x+u \in s_{h} \\
& \quad x \in s_{h} \\
& \leq \mathrm{k}_{0} \mathrm{~K}_{3} \mathrm{~h}^{\mathrm{m}-2}\|\mathrm{u}\|_{\mathrm{m}}, 2 \leq \mathrm{m} \leq \mathrm{r+1} .
\end{aligned}
$$


For $n \varepsilon \stackrel{0}{H}^{2},\|n\| \leq\left\|n_{x}\right\|$ and $\left\|n_{x}\right\| \leq\left\|n_{x x}\right\|$. Hence the result (4.16) for $j=2$. Similarly, we get the estimate $(4.16)$, for $j=0$, consequently, the estimate for $\|n\|_{1}$ follows from the interpolation inequality,

$$
\|n\|_{1} \leq\|n\|^{1 / 2}\|n\|_{2}^{1 / 2}
$$

In order to estimate $n_{t}$ ', we differentiate $(4.5)$ with respect to ' $t$ ' and obtain

$$
A_{\rho}\left(u ; \eta_{t}, x\right)=-\left(\left[\frac{d a}{d t}(u)\right] \eta_{x x}, x_{x x}\right)+u_{t x}(1)\left(x \eta_{x}, x_{x x}\right)
$$

Identifying the right hand side of (4.18) with $F(X)$, we see that for $\phi \varepsilon H^{2}(I)$

$$
|F(\phi)| \leq k_{6}\left\|\eta_{x x}\right\|\left\|\phi_{x x}\right\|,
$$

where $k_{6}$ depends on $k_{1}$ and $\left\|u_{t}\right\|_{W^{1, \infty}}$.

Further, for $\phi \in \mathrm{D}\left(\mathrm{L}^{\star}\right)$ and $\mathrm{u} \in \mathrm{O}^{2}$, we get on integration by parts

$$
\begin{aligned}
F(\phi) & =\left(n_{x},\left(a_{t}(u) \phi_{x x}\right)_{x}\right)-u_{t x}(1)\left(n,\left(x \phi_{x x}\right){ }_{x}\right) \\
& =-\left(n,\left(a_{t}(u) \phi_{x x}\right)_{x x}\right)+u_{t x}(1)\left(n,\left(x \phi_{x x}\right)_{x}\right)
\end{aligned}
$$

and

$$
|F(\phi)| \leq k_{7}|| n\|\| \phi \|_{4}
$$

where $k_{7}=k_{7}\left(k_{1},\left\|u_{t x x}\right\|_{L}^{\infty},\left\|u_{x x}\right\|_{L}\right.$ and $\left.\left\|u_{t}\right\|_{W^{1, \infty}}\right)$.

Thus, Lemma 4.2 is applicable with $M_{1}=k_{6}\left\|n_{x x}\right\|$ and $M_{2}=K_{7}\|n\|$ and we get the desired estimate (4.17) for $j=0,2$. For $j=1$, as usual we make use of the interpolation inequality. We shall also need later the following estimate for $n_{x}(1)$.

LEMA 4.4. There is a constant $K_{8}=K_{8}\left(\alpha, K_{0}, M ; K_{4}\right)$ such that for $2 \leq m \leq r+1$.

$$
\left|n_{x}(1)\right| \leq k_{8} h^{2(m-2)}\|u\|_{m}
$$

PROOF. Define an auxiliary function $\phi \varepsilon \mathrm{H}^{4} \quad \stackrel{0}{\mathrm{H}^{2}}$ as a solution of

$$
\begin{aligned}
& L^{*}(u) \phi=0, X \varepsilon I \\
& \left.\phi_{X X X}\right|_{X=0}=0 ; \\
& \left.\phi_{X X}\right|_{X=1}=1 .
\end{aligned}
$$


Multiplying by $n$ the first equation and integrating by parts, we obtain

$$
\begin{aligned}
\alpha\left|\eta_{x}(1)\right| & \leq\left|a(0) \eta_{x}(1)\right| \leq\left|A_{\rho}(u ; n, \phi)\right| \\
& \leq A_{\rho}(u ; n, \phi-x), x \in S_{h} \\
& \leq M\|n\|_{2} \underset{x \in S_{h}}{\inf }\|\phi-x\|_{2} \\
& \leq\left. M_{4} K_{0} h^{2(m-2)}|| u\right|_{m}\|\phi\|_{m}
\end{aligned}
$$

Hence, the result follows.

\section{A PRIORI ERROR ESTIMATES GOR CONTINUOUS TIME GALERKIN APPROXIMATION.}

Throughout this section, we assume that there are positive constants $K^{*}$ and $h_{0}$ such that a Galerkin approximation $u^{h} \varepsilon S_{h}$ in (3.2) exists and satisfies,

$$
\left\|\mathrm{u}^{\mathrm{h}}\right\|_{\mathrm{K}^{\infty}\left(\mathrm{H}^{2}\right)} \leq \mathrm{K}^{\star}, \text { for } 0<\mathrm{h} \leq \mathrm{h}_{0},
$$

where $u^{h}(x, 0)$ is defined as $Q_{h} g$, satisfying

$$
A_{\rho}\left(g ; g-Q_{h} g, x\right)=0, x \varepsilon \stackrel{0}{S}_{h^{\circ}}
$$

Clearly, $\quad u^{h}(x, 0) \equiv \tilde{u}(x, 0)$.

$$
\text { Let } \zeta=u^{h}-\tilde{u} \text { and } e=u-u^{h}=\eta-\zeta \text {. }
$$

THEOREM 5.1. Suppose $n=u-\tilde{u}$ satisfies (4.5) and $u^{h}$, the Galerkin approximation of $u$ is defined by (3.2) with $Q_{h}$ given as in (5.2). Further, assume that (5.1) holds. Then, there is a constant $K_{9}=K_{9}\left(\alpha, \rho, K^{\star}, K_{1}, K_{4}, K_{5}\right.$ and $\left.K_{8}\right)$ such that for $m \geq 4$

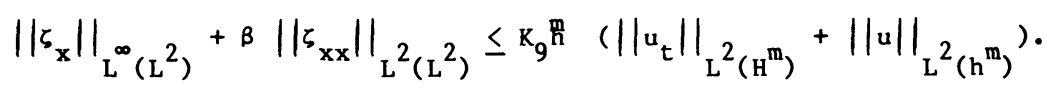

PROOF. From (4.5) and (3.1) with $v=x$, we get

$$
\left(\tilde{u}_{t x}, x_{x}\right)+A_{\rho}(u ; \tilde{u}, x)=-\left(n_{t x}, x_{x}\right)+\rho\left(u_{x}, x_{x}\right), x \varepsilon s_{h} \cdot
$$

Subtracting this from (3.2), we obtain

$$
\begin{gathered}
\left(\zeta_{t x}, x_{x}\right)+A_{\rho}\left(u^{h} ; u^{h}, x\right)-A_{\rho}(u ; \tilde{u}, x)=\left(n_{t x}, x_{x}\right)-\rho\left(\eta_{x}, x_{x}\right) \\
+\rho\left(\zeta_{x}, x_{x}\right)
\end{gathered}
$$


But

$$
\begin{aligned}
& A_{\rho}\left(u^{h} ; u^{h}, x\right)-A_{\rho}(u ; \tilde{u}, x)=\left(a\left(u^{h}\right) \zeta_{x x}, x_{x x}\right) \\
& +\left(\left[a\left(u^{h}\right)-a(u)\right] \tilde{u}_{x x}, x_{x x}\right)-u_{x}(1)\left(x \zeta_{x}, x_{x x}\right)+\eta_{x}(1)\left(x u_{x}^{h}, x_{x x}\right) \\
& \quad-\zeta_{x}(1)\left(x_{x}^{h}, x_{x x}\right)+\rho\left(\zeta_{x}, x_{x}\right)
\end{aligned}
$$

From (5.4)-(5.5) with $x=\zeta$, it follows on integrating by parts with respect to $x$ the two terms on the right hand side of (5.4),

$$
\begin{aligned}
\frac{1}{2} \frac{d}{d t}\left\|\zeta_{x}\right\|^{2} & +\left(a\left(u^{h}\right) \zeta_{x x}, \zeta_{x x}\right)=-\left(\eta_{t}, \zeta_{x x}\right)+\rho\left(\eta, \zeta_{x x}\right)+u_{x}(1)\left(x \zeta_{x}, \zeta_{x x}\right) \\
& +\left(\left[a(u)-a\left(u^{h}\right)\right] \tilde{u}_{x x}, \zeta_{x x}\right)-\eta_{x}(1)\left(x_{x} h, \zeta_{x x}\right)+\zeta_{x}(1)\left(x_{x} u_{x}, \zeta_{x x}\right)
\end{aligned}
$$

Using $a(\cdot) \geq \alpha,(5.1)$ and replacing $\tilde{u}$ by $u-n$, we obtain

$$
\begin{aligned}
& \frac{1}{2} \frac{d}{d t}\left\|\zeta_{x}\right\|^{2}+\alpha\left\|\zeta_{x x}\right\|^{2} \leq\left\{\left\|\eta_{t}\right\|+\rho\|n\|+k_{2}\left\|\zeta_{x}\right\|+k_{2}\left(\|n\|_{L}^{\infty}+\|\|_{L}^{\infty}\right)\right. \\
& \left.\|n\|_{2}+k_{1} K_{2}(\|n\|+\|\zeta\|)+K^{*}\left|n_{x}(1)\right|\right\}\left\|\zeta_{x x}\right\|+k^{*}\left|\zeta_{x}(1)\right|\left\|\zeta_{x x}\right\| .
\end{aligned}
$$

Since $\left|\zeta_{x}(1)\right| \leq\left\|\zeta_{x}\right\|^{1 / 2}\left\|\zeta_{x x}\right\|^{1 / 2}$ for $\zeta \varepsilon H^{2}$, applying Young's inequality for the last term and the inequality $a b \leq \frac{a^{2}}{2 \varepsilon}+\frac{\varepsilon}{2} b^{2}, a, b \geq 0 ; \varepsilon>0$ for the remaining terms in (5.6), we get using $\|\phi\|_{L} \leq\left\|\phi_{x}\right\|$ for $\phi \varepsilon \mathrm{H}^{2}$

$$
\begin{aligned}
\frac{1}{2} \frac{d}{d t}\left\|\zeta_{x}\right\|^{2} & +\alpha\left\|\zeta_{x x}\right\|^{2} \leq K_{10}(\varepsilon)\left\|\zeta_{x x}\right\|^{2}+K\left(K_{1}, K_{2}, K^{\star}, \rho ; \varepsilon\right)\left(\left\|n_{t}\right\|^{2}\right. \\
& \left.+\|n\|^{2}+\left|\eta_{x}(1)\right|^{2}+\left.\|n\|_{1}^{2}\|n\|\right|_{2} ^{2}\right) \\
& +K\left(K_{2} ; \varepsilon\right)\|n\|_{2}^{2}\left\|\zeta_{x}\right\|^{2}+K\left(K_{1}, K_{2} K^{\star}, \varepsilon\right)\left\|\zeta_{x}\right\|^{2}
\end{aligned}
$$

Now with appropriate choice of $\varepsilon, K_{10}(\varepsilon)$ can be made less than or equal to $\alpha / 2$. With this choice of $\varepsilon$, we get by integrating with respect to ' $t$ ' and using Gronwall's inequality the following

$$
\begin{aligned}
\left\|\zeta_{x}\right\|^{2}(t)+\alpha \int_{0}^{t}\left\|\zeta_{x x}\right\|^{2} d t^{\prime} \leq k\left(k_{1}, k_{2}, k^{*} ; \rho\right) & \int_{0}^{t}\left(\left\|n_{t}\right\|^{2}+\|n\|^{2}\right. \\
& \left.+\left|n_{x}(1)\right|^{2}+\left.\|n\|_{1}^{2}\|n\|\right|_{2} ^{2}\right) d t^{\prime} .
\end{aligned}
$$


From $(4.16)-(4.17)$ and (4.19) with $2(\mathrm{~m}-2) \geq \mathrm{m}$ and $2 \mathrm{~m}-3 \geq \mathrm{m}$ that is $\mathrm{m} \geq 4$, we get the desired estimate (5.3).

COROLLARY 5.2. Let all the assumtions of the previous theorem hold and the finite dimensional subspace $\mathrm{S}_{h}$ satisfy the inverse property. Then there is a constant $K_{11}$ depending on $K_{9}$ and $K_{0}$ such that for $r+1 \geq m \geq 4$,

$$
\begin{aligned}
\|r\|_{L^{\infty}\left(L^{2}\right)}+ & \|r\|_{L\left(H^{1}\right)}+h|| r \|_{L}^{\infty}\left(H^{2}\right) \\
& \leq \mathrm{K}_{11} h^{m}\left(\|\mathrm{u}\|_{L^{2}\left(H^{m}\right)}+\left\|u_{t}\right\|_{L^{2}\left(H^{m}\right)}\right) .
\end{aligned}
$$

PRO0F. From the estimate (5.3) and $\|\zeta\| \leq\left\|\zeta_{\mathbf{x}}\right\|$ for $\zeta \varepsilon \mathrm{s}_{h}$, we get

$$
\|\zeta\|_{L^{\infty}\left(L^{2}\right)}+\|\zeta\|_{L\left(H^{\infty}\right)} \leq k_{11} h^{m}\left(\|u\|_{L^{2}\left(H^{m}\right)}+\left\|u_{t}\right\|_{L^{2}\left(H^{m}\right)}\right) .
$$

By inverse property for $\mathrm{S}_{h}$, we have

$$
\|\zeta\|_{L}^{\infty}\left(H^{2}\right) \leq K_{0} h^{-1}\|\zeta\|_{L}^{\infty}\left(H^{1}\right), \quad \zeta \varepsilon S_{h}
$$

Hence the result (5.7). From Theorem 5.1, Corollary 5.2, Lemma 4.3 and triangle inequality we get the following theorem.

THEOREM 5.3. Let the solution $u$ of $(2.7)-(2.9)$ satisfy the regularity condition $R_{1}$. Further, suppose that there are positive constants $h_{0}$ and $K^{*}\left(K^{\star} \geq 2 K_{2}\right)$ such that an approximate solution $u^{h} \varepsilon S_{h}$ of (3.2) satisfying (5.1) exists in $I \times(0, T]$ for $0<h \leq h_{0}$. Then, the following estimates hold for $r \geq 3$,

$$
\|e\|_{L}^{\infty}\left(H^{j}\right) \leq k_{1} h^{r+1-j}, \quad j=0,1,2,
$$

where $K_{12}=K_{12}\left(K_{4}, K_{11}\right.$ and $\left.k_{2}\right)$. Besides, for sufficiently small $h$ and $r \geq 3$,

$$
\left\|\mathrm{u}^{\mathrm{h}}\right\|_{\mathrm{L}\left(\mathrm{H}^{2}\right)} \leq 2 \mathrm{~K}_{2} \leq \mathrm{K}^{*}
$$

and consequent1y, $K_{12}$ can be choosen independent of $K^{\star}$.

PRO0P. The estimates (5.8) for $j=0,1,2$ are immediate from the Theorem 5.1, Corollary 5.2 and Lemma 4.3 by triangle inequality. To prove (5.9), we note

$$
\begin{aligned}
\left\|u^{h}\right\|_{L\left(H^{2}\right)} & \leq\|u\|_{L_{\left(H^{2}\right)}^{\infty}}+\|e\|_{L\left(H^{2}\right)} \\
& \leq K_{2}+k_{12} h^{r-1} \\
& \leq 2 K_{2}, \text { for sufficiently sma11 } h \text { and } r \geq 3 .
\end{aligned}
$$


We are now looking for approximations of $U$ and $S$, where the pair $\{U, S\}$ is the solution of (2.1)-(2.4). The Galerkin approximations $U^{h}$ and $S_{h}$ are given by

$$
\begin{aligned}
U^{h}(y, \tau) & =u^{h}(x, t) \\
S^{h}(\tau) & =s_{h}(t)
\end{aligned}
$$

where,

$$
\begin{aligned}
& y=s_{h}(t) x, \\
& \tau=\tau_{h} .
\end{aligned}
$$

and $s_{h}, \tau_{h}$ are defined by $(3.4),(3.5)$ respectively.

THEOREM 5.4. Suppose that the condition $B$ and the regularity condition $\tilde{R}_{1}$ are satisfied. Then the following estimates hold for $r \geq 3$,

$$
\begin{aligned}
& || s-s_{h} \|_{L}^{\infty}\left(0, T_{0}\right) \\
& \left\|\tau-\tau_{h}\right\|_{L}^{\infty}\left(0, T_{0}\right) \\
& || \tau\left(h^{r}+1\right)
\end{aligned}
$$

and

$$
|| U-\left.U^{h}\right|_{L\left(0, T_{0} ; H^{j}(\tilde{\Omega}(\tau))\right)}=0\left(h^{r+1-j}\right), j=0,1,2,
$$

where $\|\cdot\|$ is interpreted as

$$
\|\phi\|_{L}^{\infty}\left(0, \mathrm{~T}_{0} ; \mathrm{H}^{\mathrm{j}}(\tilde{\Omega}(\tau))\right)=\int_{0}^{\mathrm{T}}\|\phi\|_{\mathrm{H}^{\mathrm{j}}(\tilde{\Omega}(\tau))} \mathrm{d \tau}
$$

with $\tilde{\Omega}(\tau)=\left(0, \min \left(S(\tau), S_{h}(\tau)\right)\right)$.

PROOF. From $(2.10)$ and $(3.4)$, we have

$$
\left|s-s_{h}\right| \leq \int_{0}^{t}\left(\left|n_{x}(1)\right|+\left|\zeta_{x}(1)\right|\right)|s| d t^{\prime}+\int_{0}^{t}\left|u_{x}^{h}(1)\right|\left|s-s_{h}\right| d t^{\prime} \cdot
$$

An application of Gronwa11's inequality and the estimates $(4.19),(5.3)$, for $m=r+1$ gives

$$
\begin{aligned}
& \left\|s-s_{h}\right\|_{L(0, T)}^{\infty} \leq k\left(K_{2}\right)\left\{\left\|\eta_{x}(1)\right\|_{L^{2}(0, T)}+\left\|\zeta_{x}(1)\right\|_{L^{2}(0, T)}\right\} \\
& \leq \mathrm{K}\left(\mathrm{K}_{2}, \mathrm{~K}_{8}\right)\left\{\mathrm{h}^{2(\mathrm{r}-1)}|\mathrm{u}|_{\mathrm{L}^{2}\left(\mathrm{H}^{\mathrm{r}+1}\right)}+|| \zeta_{\mathrm{xx}} \|_{\mathrm{L}^{2}\left(\mathrm{~L}^{2}\right)}\right\} \\
& \leq \mathrm{K}_{13} \mathrm{~h}^{\mathrm{r}+1}, \text { for } \mathrm{r} \geq 3
\end{aligned}
$$


The estimate $(5.13)$ is immediate from (5.16), if we note that

$$
\left\|s-s_{h}\right\|_{L\left(0, T_{0}\right)}=\left\|s-s_{h}\right\|_{L(0, T)} .
$$

Further, the estimate (5.14) follows from (2.11), (3.5) and (5.16). Finally since

$$
\left\|U-U^{h}\right\|_{L}^{\infty}\left(0, T_{0} ; H^{j}(\tilde{\Omega}(\tau))\right) \leq\left\|u-u^{h}\right\|_{L\left(0, T ; H^{j}(I)\right)}
$$

we obtain the required estimate (5.15).

6. GLOBAL EXISTENCE AND UNIQUENESS OF THE GALERKIN APPROXIMATION.

Now we consider the problem of existence of the Galerkin approximation $\mathbf{u}^{\mathrm{h}}$ in the domain of existence of $u$. Towards this, let us recall (5.4) and note

$$
\begin{aligned}
A_{\rho}\left(u^{h} ; u^{h}, x\right)-A_{\rho}(u ; \tilde{u}, x)= & A_{\rho}(u ; \zeta, x)+\left(\left[a\left(u^{h}\right)-a(u)\right] u_{x x}^{h}, x_{x x}\right) \\
& +\eta_{x}(1)\left(x u_{x}^{h}, x_{x x}\right)-\zeta_{x}(1)\left(x u_{x}^{h}, x_{x x}\right) .
\end{aligned}
$$

From the above, we get

$$
\begin{gathered}
\left(\zeta_{t x}, x_{x}\right)+A_{p}(u ; \zeta, x)=\left(n_{t x}, x_{x}\right)-\rho\left(\eta_{x}, \zeta_{x}\right)+\rho\left(\zeta_{x}, x_{x}\right)+\left(\left[a(u)-a\left(u^{h}\right)\right]\right. \\
\left.u_{x x}^{h}, x_{x x}\right)-\eta_{x}(1)\left(x u_{x}^{h}, x_{x x}\right)+\zeta_{x}(1)\left(x u_{x}^{h}, x_{x x}\right)
\end{gathered}
$$

But,

$$
a(u)-a\left(u^{h}\right)=\tilde{a}_{u} e=-\int_{0}^{1} \frac{\partial a}{\partial u}(u-\xi e) \text { e d } \xi
$$

Replacing $u^{h}$ by $u-e$ in (6.1) with $(6.2)$, we have

$$
\begin{aligned}
& \left(\zeta_{t x}, x_{x}\right)+A_{\rho}(u ; \zeta, x)=-\left(n_{t}, x_{x x}\right)+\rho\left(n, x_{x x}\right)+\rho\left(\zeta_{x}, x_{x}\right) \\
& -\left(\int_{0}^{1} \frac{\partial a}{\partial u}(u-\xi e)(n-\zeta) d \xi\left(u_{x x}-e_{x x}\right), x_{x x}\right)-\eta_{x}(1)\left(x\left(u_{x}-e_{x}\right), x_{x x}\right) \\
& +\zeta_{x}(1)\left(x\left(u_{x}-e_{x}\right), x_{x x}\right) \text {. }
\end{aligned}
$$

Substitute $e$ by $E(x, t)$, where $E \varepsilon H^{2}$. Then we get

$$
\begin{aligned}
\left(\zeta_{t x}, x_{x}\right) & +A_{\rho}(u ; \zeta, x)=-\left(n_{t}, x_{x x}\right)+\rho\left(\eta, x_{x x}\right)+\rho\left(\zeta_{x}, x_{x}\right) \\
& -\left(\int_{0}^{1} \frac{\partial a}{\partial u}(u-\xi E)(n-\zeta) d \xi\left(u_{x x}-E_{x x}\right), x_{x x}\right)-\eta_{x}(1)\left(x\left(u_{x}-E_{x}\right), x_{x x}\right) \\
& +\zeta_{x}(1)\left(x_{\left.\left(u_{x}-E_{x}\right), x_{x x}\right)}\right.
\end{aligned}
$$


which is a linear ordinary differential equation in $\zeta$. Therefore, for any $E=E(x, t)$ there exists a unique solution $\zeta$ of (6.3) with

$$
\zeta(\mathrm{x}, 0)=0
$$

in the interval $(0, \mathrm{~T}]$.

The equation (6.3) defines an operator $J$ such that $\zeta=J(E)$, for each E $\varepsilon \mathrm{O}^{2}$. Since $\mathrm{e}=n-\zeta$, therefore

$$
e=n-\mathcal{J}(E), \text { for each } E \in \stackrel{0}{H}^{2} \text {. }
$$

To show the existence of a solution $u^{h}$ of (3.2), we need to show that the operator equation (6.5) has a fixed point. In other words, we are looking for an e(E) such that

$$
e(E)=E
$$

THEOREM 6.1. Suppose that the finite dimensional space $\mathrm{S}_{\mathrm{h}}$ satisfies inverse property and $u$ is the unique solution of (2.7)-(2.9). Further, let the regularity conditions $R_{1}$ be satisfied. Then for some $\delta>0$, there exists a solution $u^{h} \varepsilon S_{h}$ of (3.2) satisfying $\left\|\mathrm{u}-\mathrm{u}^{\mathrm{h}}\right\|_{\mathrm{L}\left(0, \mathrm{~T}_{0} ; \mathrm{H}^{2}(\mathrm{I})\right)} \leq \delta$.

PROOF. Set $x=\zeta$ in (6.3) to get

$$
\begin{aligned}
& \frac{1}{2} \frac{d}{d t}\left\|\zeta_{x}\right\|^{2}+\tilde{\alpha}\left\|\zeta_{x x}\right\|^{2} \\
& \leq\left\{\left\|n_{t}\right\|+\rho\|n\|+k_{1}\left(\|n\|_{L}^{\infty}+\|\zeta\|_{L}\right)\left(\left\|u_{x \mathbf{x}}\right\|+\left\|E_{x \mathbf{x}}\right\|\right)\right. \\
& \left.+\left|n_{x}(1)\right|\left(\left\|u_{x}\right\|+\left\|E_{x}\right\|\right)\right\}\left\|\zeta_{x x}\right\|+\rho\left\|\zeta_{x}\right\|^{2} \\
& +\left(\left\|u_{x}\right\|+\left\|E_{x}\right\|\right)\left|\zeta_{x}(1)\right|\left\|\zeta_{x x}\right\|
\end{aligned}
$$

Using $\left|\zeta_{\mathbf{x}}(1)\right| \leq\left\|\zeta_{\mathbf{x}}\right\|^{1 / 2}\left\|\zeta_{\mathbf{x x}}\right\|^{1 / 2}$, applying Young's inequality for the 1ast term and $a b \leq \frac{a^{2}}{2 \varepsilon}+\frac{\varepsilon}{2} b^{2}, a, b \geq 0 ; \varepsilon>0$ for the remaining terms, we get

$$
\begin{aligned}
& \frac{\mathrm{d}}{\mathrm{dt}}\left\|\zeta_{\mathbf{x}}\right\|^{2}+2 \tilde{\alpha}\left\|\zeta_{\mathbf{x x}}\right\|^{2} \\
& \leq \mathrm{K}_{14}(\varepsilon)\left\|\zeta_{\mathrm{xx}}\right\|^{2}+\mathrm{K}\left(\mathrm{K}_{1}, \mathrm{~K}_{2}, \rho ; \varepsilon\right)\left\{|| n_{\mathrm{t}} \|^{2}+\left(\|n\|_{\mathrm{L}}^{2}\right.\right. \\
& \left.+\left|\eta_{x}(1)\right|\right)\left(1+|| E||_{2}^{2}\right\}+k\left(K_{1}, K_{2}, \rho ; \varepsilon\right)\left(1+\left.|| E\right|_{2} ^{2}\right)\left\|\zeta_{x}\right\|^{2}
\end{aligned}
$$

Choosing $\varepsilon$ appropriately so that $2 \tilde{\alpha}=\mathrm{K}_{14}(\varepsilon)$, integrating with respect to ' $t$ ' and there after applying Gronwall's inequality, we get 


$$
\begin{aligned}
& \|\zeta\|_{1}^{2}(t) \leq K\left(K_{1}, K_{2} ; \rho\right) \exp \left[K ( \rho , K _ { 1 } ; K _ { 2 } ) t ( 1 + \| E \| _ { L } ^ { \infty } ( H ^ { 2 } ) ] \int _ { 0 } ^ { t } \left\{\left\|n_{t}\right\|^{2}\right.\right. \\
& +\left(\|n\|_{1}^{2}+\left|n_{x}(1)\right|^{2}\right)\left(1+\|E\|_{L}^{2}\left(H^{2}\right)\right\} .
\end{aligned}
$$

From the estimates $(4.16),(4.17)$ and $(4.19)$, it follows that

$$
\left.\|\zeta\|_{L^{\infty}\left(H^{1}\right)} \leq K_{15^{2}} h^{r+1}+\left(h^{r}+h^{2(r-1)}\right)\left(1+|| E \|_{L^{\infty}\left(H^{2}\right)}^{2}\right)\right\},
$$

where $\mathrm{K}_{15}=\mathrm{K}_{15}\left(\mathrm{~K}_{1}, \mathrm{~K}_{2}, \mathrm{~K}_{4}, \mathrm{~K}_{8}, \rho\right.$ and $\left.\|\mathrm{E}\|_{\mathrm{L}\left(\mathrm{H}^{2}\right)}\right)$. Thus we have

$$
\begin{aligned}
\|e\|_{L^{\infty}\left(H^{2}\right)} & \leq\|n\|_{L^{\infty}\left(H^{2}\right)}+\|\zeta\|_{L^{\infty}\left(H^{2}\right)} \\
& \leq\|n\|_{L^{\infty}\left(H^{2}\right)}+K_{0^{H}}{ }^{-1}\|\zeta\|_{L^{\infty}\left(H^{1}\right)} \cdot
\end{aligned}
$$

For $\|E\|_{L}^{\infty}\left(H^{2}\right) \leq \delta$ and from $(4.16),(6.6),(6.7)$, we get

$$
\|\mathrm{e}\|_{L\left(\mathrm{H}^{2}\right)} \leq \mathrm{K}_{16} \mathrm{~h}^{\mathrm{r}-1} \text {, where } \mathrm{K}_{16}=\mathrm{K}_{16}\left(\mathrm{~K}_{15}, \mathrm{~K}_{4}, \mathrm{~K}_{0} ; \delta\right) .
$$

Therefore, for sufficiently small $h$

$$
\|\mathrm{e}\|_{L^{\infty}\left(H^{2}\right)} \leq \delta
$$

Now, an application of Schauder's fixed point theorem guarantees the existence of an $E$ such that $\mathbf{e}=\mathrm{E}$, which is a solution of the operator equation (6.5). The uniqueness of the approximate solution $u^{h}$ is easy to prove. So we formalize the above in the form of a Theorem.

THEORBM 6.2. Let all the hypotheses of the Theorem 6.1 be satisfied and let $K>0$. Then there exists one and only one solution $u^{h} \varepsilon S_{h}$ of (3.2) in the ball $\left\{\left\|u-u^{h}\right\|_{L\left(H^{2}\right)} \leq k\right\}$, for sufficiently small $h$ and $r \geq 3$. 


\section{REPBRENCES}

1. NITSCHE, J.A. Finite Element Approximations to the One Dimensional Stefan Problem, Proc. on Recent Advances in Numerical Analysis, C. de Boor and G. Golub eds., Academic Press, NY (1978), 119-142.

2. NITSCHE, J.A. A Finite Element Method for Parabolic Free Boundary Problems, Inst. Naz. di Alta Matematica, Vo1. I (M. Magenes ed.) (1980), 277-318.

3. NITSCHE, J.A. Finite Element Approximation to One Phase Stefan Problem, Free Boundary Problems: Theory and Appl. an Interdisciplinary Symp., Motecantini, (Italy) Vol. II (Research Notes in Math. 79), Pitman Adv. Publ. Program. Boston, London and Melbourne (1983).

4. MAGEnES, E. Topics in Parabolic Equations: Some Typical Free Boundary Problems, Boundary Value Problems for Linear Partial Differential Equations, Proc. NATO Adv. Stud. Inst. Liege, Belgium, Sept. 1976, H.G. Garnier ed., D. Reidel Pub1. Co., Dortrecht (1976), 239-312.

5. DAS, P.C. and PANI, A.K. A Priori Error Estimates in $\mathrm{H}^{1}$ and $\mathrm{H}^{2}$-norms for Galerkin Approximations to a Single Phase Nonlinear Stefan Problem, (Submitted).

6. DAS, P.C. and PANI, A.K. A Galerkin Method For a Single Phase Nonlinear Stefan Problem with Dirichlet Boundary Conditions, (Submitted).

7. FASANO, A. and PRIMICERIO, M. Free-Boundary Problems For Nonlinear Parabolic Equations With Non1inear Free Boundary Conditions, J. Math. Ana1. App1. 72 (1979), 247-273.

8. LANDAU, H.A. Heat Conduction in Melting Solid, Quart. J. App1. Math. 8 (1950), $81-94$

9. ODEN, J.T. and REDDY, J.N. An Introduction To the Mathematical Theory of Finite Elements, Wiley Interscience, NY (1976). 


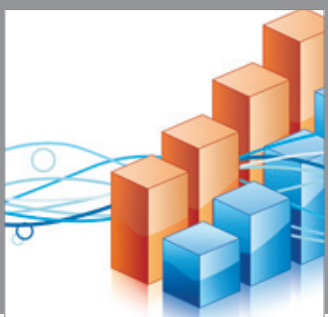

Advances in

Operations Research

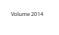

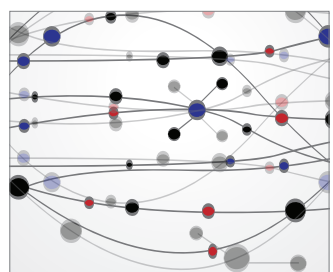

\section{The Scientific} World Journal
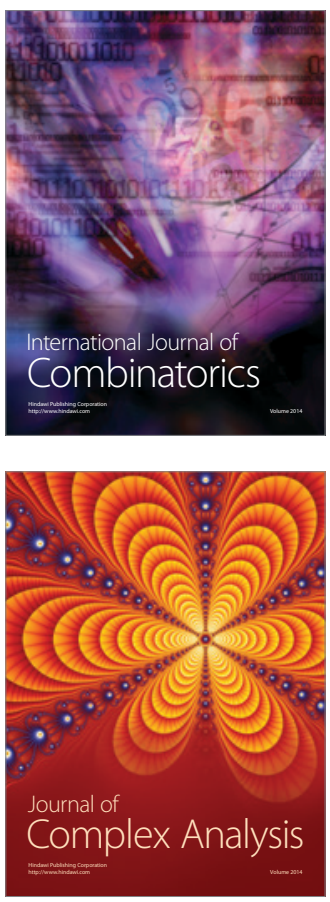

International Journal of

Mathematics and

Mathematical

Sciences
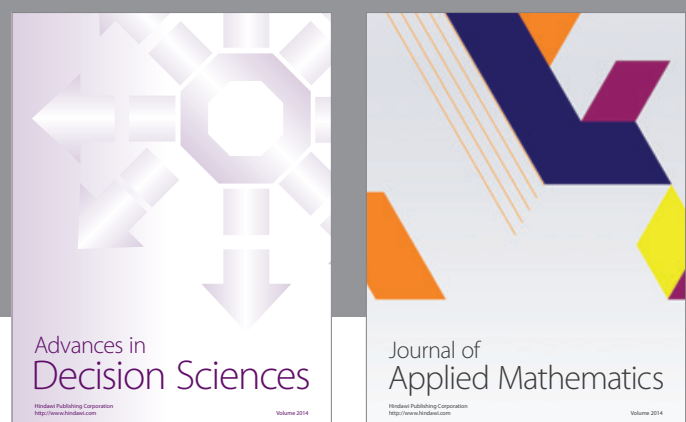

Journal of

Applied Mathematics
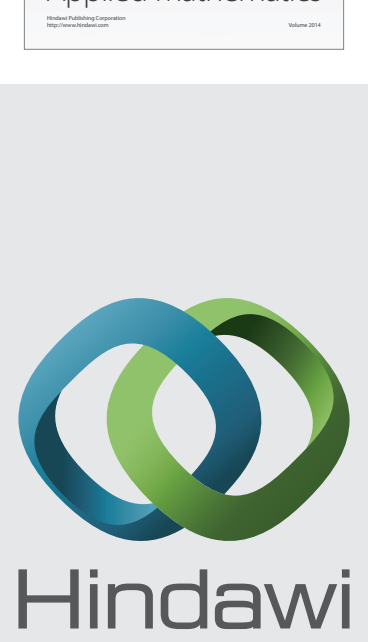

Submit your manuscripts at http://www.hindawi.com
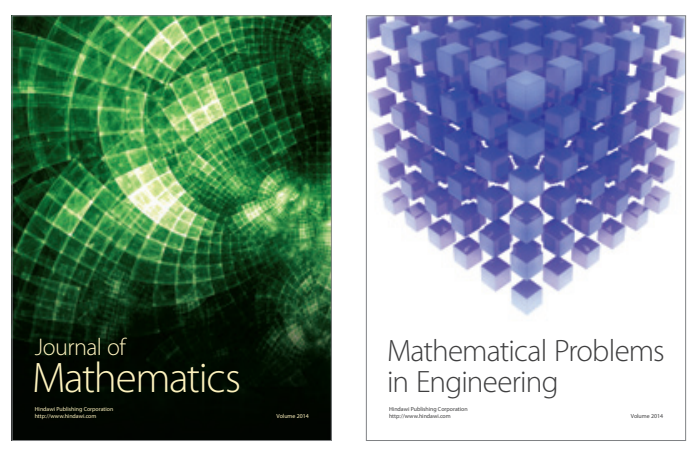

Mathematical Problems in Engineering
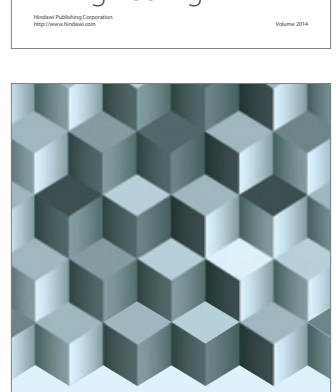

Journal of

Function Spaces
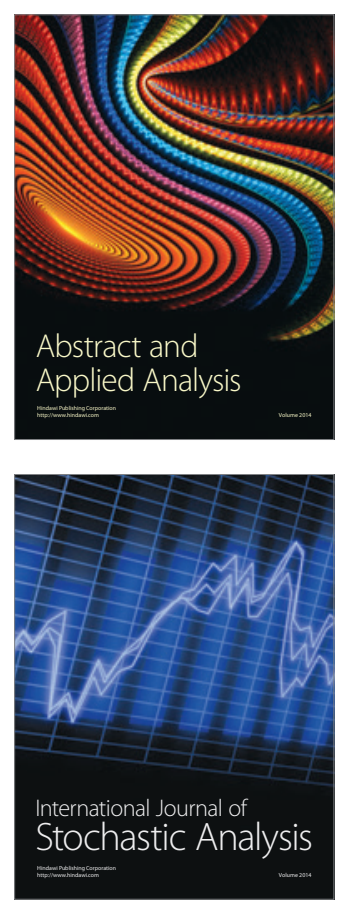

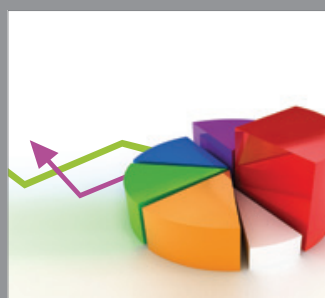

ournal of

Probability and Statistics

Promensencen
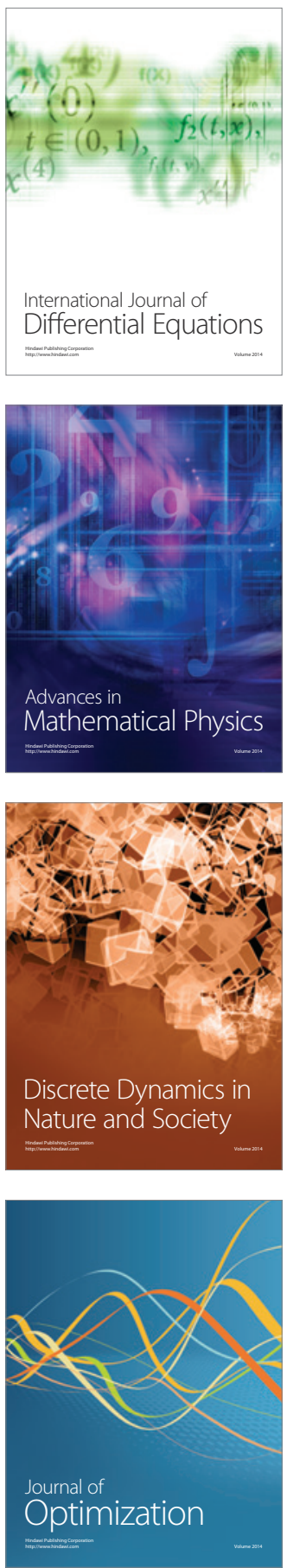